\title{
Aspectos farmacológicos do anticorpo monoclonal (Nivolumab) utilizando a Via PD-1 no tratamento antitumoral do Linfoma de Hodgkin.
}

\author{
Pharmacological aspects of the monoclonal antibody (Nivolumab) using \\ the PD-1 Pathway in antitumor treatment of Hodgkin's Lymphoma.
}

Recebido em: 27/02/2018 Aceito em: 15/01/2019
Fernanda Alves da SILVA; Alessandra STROKA; Viviane FUSCO Universidade Paulista, Campus Sorocaba. Av. Independência, 210, Éden, CEP 18087-

101. Sorocaba, SP, Brasil.E-mail:fernanda.mg.alves@hotmail.com

\section{ABSTRACT}

Hodgkin's disease, more commonly referred to Hodgkin's lymphoma is a type of cancer that affects the lymphatic system, lymph nodes which are defense cells of the organism. Hodgkin's Lymphoma (LH) has the Reed-Sternberg cells (RS), with immune tolerance, able to avoid the immune system recognition, since they have a state of non-specific reactivity, which results in the absence of response to antigens of the body itself. It is believed that the ability of tumor cells to avoid the action of the immune system is due to the super-expressão of the 9p24 gene, thereby increasing the protein-encoding PD-L1, which interacts with the PD-1 receptor on T cells, promoting a sign immunosuppression, which allows the tumor cell is not affected by the T lymphocyte Nivolumab, approved by the FDA in 2014 and by Anvisa in 2018, it is part of a class of drugs targeting the release of control points of the immune system that regulate the antitumor response. This drug acts by inhibition of PD-1 receptor on T cells, blocking this interaction with proteins PD-L1 and PDL2. Thus, this research it is a descriptive review of the literature and aimed to gather published information related to the use of immunotherapy with Nivolumab in order to identify their pharmacological actions, as well as adverse effects more frequent produced by this drug. From that survey concluded that Nivolumab therapy is promising since there are numerous reports of cases of remission of the tumor with fewer side effects when compared to standard therapy.

Keywords: Hodgkin's lymphoma; Nivolumab; monoclonal antibody; way PD-1/PD-L1.

\section{RESUMO}

A doença de Hodgkin, mais comumente chamada de Linfoma de Hodgkin é um tipo de câncer que acomete os gânglios do sistema linfático, nos quais se encontram células de defesa do organismo. O linfoma de Hodgkin (LH) possui as células de Reed-Sternberg (RS), portadoras de tolerância imunológica, capazes de evitar o reconhecimento do sistema imunológico, uma vez que apresentam um estado de não-reatividade específica, o que resulta em ausência de resposta a antígenos do próprio organismo. Acredita-se que a capacidade das células tumorais de evitar a ação do sistema imunológico é decorrente da super-expressão do gene 9p24, aumentando assim a codificação da proteína PD-L1, que interage com o receptor PD-1 nos linfócitos $\mathrm{T}$, promovendo um sinal de imunossupressão, o que permite que a célula tumoral não seja atingida pelo linfócito T. O Nivolumab, aprovado pela FDA em 2014 e pela Anvisa em 2018, faz parte de uma classe de medicamentos direcionados à liberação de pontos de controle do sistema imunológico que regulam a 
resposta antitumoral. Esse fármaco atua na inibição de receptor PD-1 nos linfócitos T, bloqueando a interação deste com as proteínas PD-L1 e PD-L2. Dessa forma, esta pesquisa trata-se de uma revisão descritiva da literatura e teve por objetivo reunir informações publicadas relacionadas ao uso de imunoterapia com Nivolumab, a fim de identificar suas ações farmacológicas, bem como os efeitos adversos mais frequentes produzidos por esse fármaco. A partir desse levantamento concluiu-se que a terapia com Nivolumab é promissora, uma vez que há inúmeros relatos de casos de remissão do tumor com menos efeitos colaterais quando comparados à terapia clássica.

Palavras-chave: Linfoma de Hodgkin; Nivolumab; anticorpo monoclonal; Via PD-1/PD-L1.

\section{INTRODUÇÃO}

Linfomas são neoplasias malignas provocadas por defeitos em oncogenes ou genes supressores de tumores, resultando em elevado número de cópias de células tumorais do sistema imunológico. É a sexta doença maligna mais comum e pode afetar tanto linfócito $\mathrm{T}$ quanto linfócito B. Os linfomas são classificados em Linfoma não-Hodgkin e Linfoma de Hodgkin (LH), sendo o ultimo, tema deste estudo. O LH foi descrito pelo médico inglês Thomas Hodgkin em 1832 (1) e trata-se de um tipo de câncer com origem no sistema linfático, a partir de um linfócito (mais comumente do tipo B) com características malignas e elevada capacidade de multiplicação e disseminação. Essas células se multiplicam nos linfonodos, mais frequentemente na região do pescoço e mediastino, a partir dos quais podem atingir outros tecidos (2).

O LH é caracterizado por um tipo de célula cancerosa, a célula de Reed-Sternberg (RS) que na microscopia apresenta características peculiares (1). Essas células são geralmente derivadas dos linfócitos $\mathrm{B}$, e mesmo em número reduzido sofrem alteração maligna (3). Nas células RS há o gene 9p24 no cromossomo 9p que codifica PD-L1 e PD-L2 em um número muito maior do que em outros tipos células de linfoma (4-7). A partir disso, a neoplasia do tipo RS é a que possui uma clínica mais agressiva, considerando que suas células apresentam capacidade de evitar o sistema antitumoral, liberando citocinas imunossupressoras e impedindo que o linfócito $\mathrm{T}$ consiga matar a célula tumoral $(4,6)$.

A escolha do tratamento antineoplásico é determinada de acordo com o estágio da doença, podendo ser realizado com quimioterapia e radioterapia, de forma isolada ou combinadas. Os efeitos colaterais mais comuns decorrentes da quimiotera- pia consistem em náusea/vômito, flebite, mucosite, diarreia e alterações hematológicas $(8,9)$ individualized to the stage of the lymphoma permitting minimization of late toxicity such as infertility, premature menopause, cardiac disease, and most importantly, risk of second neoplasms. More than $90 \%$ of patients with limited Hodgkin's lymphoma can be cured with either short-course chemotherapy alone or even briefer chemotherapy followed by involved-field radiation. Accumulating evidence suggests that chemotherapy alone is suitable for the large majority of patients with limited disease. For the $80 \%$ of patients with advanced disease but without a large number of adverse prognostic factors, standard multi-agent chemotherapy with the well-established ABVD regimen (doxorubicin, bleomycin, vinblastine, and dacarbazine.

$\mathrm{O}$ tratamento quimioterápico do LH foi impulsionado a partir de pesquisas realizadas por DeVita e cols. em 1964, quando pacientes foram tratados com uma combinação de mostarda nitrogenada, vincristina, procarbazina e prednisona (MOPP). Esse esquema terapêutico visava aumentar o efeito antitumoral, sem aumento da toxicidade. Como resultado, essa terapia demonstrou taxa de resposta completa de $84 \%$ dos pacientes (10).

Ensaios clínicos posteriores foram realizados com uma combinação diferente de fármacos: doxorrubicina (adriamicina), bleomicina, vimblastina e dacarbazina (ABVD), resultando em redução do risco de neoplasia secundária, quando comparado ao esquema MOPP (11)bleomycin, vinblastine, and imidazole carboxamide. The purpose for designing this new combination was two-fold: to compare the efficacy of ABVD with MOPP, and to demonstrate absence of cross-resistance between the two regimens. Of 60 patients entered into the study, 45 (MOPP25, ABVD20. Por fim, em 1992, ensaios 
clínicos randomizados e multicêntricos mostraram que o esquema $A B V D$ é superior ao esquema MOPP em relação à resposta ao tratamento, sobrevida livre da doença e sobrevida global, constituindo, portanto, a terapêutica de escolha para LH em estágios avançados $(12,13)$.

Mesmo com os avanços terapêuticos, 15\% dos pacientes portadores de $\mathrm{LH}$ apresentam recidiva após o tratamento com quimioterapia clássica, sendo que a duração do período de remissão diminui a cada novo tratamento. Assim, o tratamento ideal deve induzir resposta duradoura e com a maior taxa de sobrevida (3).

O mais recente tratamento antitumoral proposto está atrelado às vias de controle imunológico ou pontos de verificação imunológicos $(3,5)$. Esses pontos de controle consistem em vias inibitórias de coestimulação, capazes de estabelecer modulação da duração e da extensão da resposta imune (5). Os tumores empregam essas vias para esquivar-se das células antitumorais específicas, causando resistência tumoral. Por serem interações do tipo ligante-receptor, essas ligações podem ser bloqueadas por anticorpos monoclonais $(14,15)$.

Desde 1970 pesquisas buscam elucidar o sistema imunológico para produzir um agente farmacológico que amplie a resposta imune antitumoral (16). O primeiro imunoterapêutico desenvolvido foi o Ipilimumab, potencializador das células $T$, que bloqueia o sinal inibitório de CTLA-4 (antígeno-4 do linfócito $\mathrm{T}$ citotóxico), regulador negativo da ativação de célula $T(5,21)$. Com o bloqueio dessa proteína o linfócito fica ativo por mais tempo, resultando em aumento do tempo de ação antitumoral $(7,9)$.

O Nivolumab foi aprovado pela Food and Drug Administration (FDA) em 2014 e é o fármaco que age na liberação de pontos de controle do sistema imunológico que regulam a resposta antitumoral, mais recentemente liberado pela Agência Nacional de Vigilância sanitaria (Anvisa) em 2018. Esse fármaco ativa linfócitos $\mathrm{T}$ inibindo o receptor PD-1 nessas células, bloqueando a interação deste com as proteínas PD-L1 e PD-L2. Estudos recentes apontam relatos de casos de remissão de LH com o emprego desse fármaco, o que motivou esta pesquisa, que teve por objetivo reunir informa- ções publicadas relacionadas ao uso de imunoterapia com Nivolumab, a fim de identificar suas ações farmacológicas no reforço do sistema imunológico com direcionamento à célula maligna, bem como os efeitos adversos mais frequentes produzidos por esse fármaco $(17,18)$.

Aspectos farmacológicos do Nivolumab. O Nivolumab ativa linfócitos $T$ por meio da inibição de receptor PD-1 nessas células, bloqueando a interação deste com as proteínas PD-L1 e PD-L2 (9). O PD-1 é um receptor expresso em macrófagos ativados, células dendríticas, células B, célula NK e células $\mathrm{T}$, principalmente ativadas, e trata-se de uma proteína que possui domínios intracelulares e extracelulares, além da porção transmembrana $(6,7)$. A principal função do PD-1 é conter a ação dos linfócitos $\mathrm{T}$ nos tecidos periféricos quando há uma resposta inflamatória, evitando danos ao hospedeiro. No entanto, a expressão dessa via também ocorre em células tumorais, fazendo com que o tumor seja capaz de evitar a resposta antitumoral $(4,6)$.

O PD-1 possui dois ligantes diferentes, o PDL1 e o PD-L2, sendo o primeiro expresso nas células T, células apresentadoras de antígenos (APC) e outras células não hematopoiéticas (incluindo células do endotélio vascular, células da ilhota pancreática e neurônios), enquanto o PD-L2 está principalmente nas células dendríticas e nos macrófagos $(3,4,12,13)$. Quando os linfócitos estão ativados, a expressão do PD-1 aumenta, o que permite aos tumores desenvolverem mecanismos, que por meio da proteína PD-L1, se liga ao linfócito e impede sua ativação (22). A célula $\mathrm{T}$ é ativada pela atividade da fosfatidil-inositol 3 quinase (PI3K) via SHIP-2 favorecendo as vias de sinalização intracelular $(14,23,24)$. A ativação PD-1 inibe essa fosforilação, impedindo que o linfócito seja ativado e promova danos aos tecidos normais. Por outro lado, no processo tumoral, o linfócito $\mathrm{T}$ permanece inativado, dando ao tumor oportunidade de progre$\operatorname{dir}(22)$.

Os anticorpos monoclonais, como o Nivolumab, bloqueiam a ligação entre PD-1 e PD-L1, produzindo respostas clínicas duráveis em vários tipos de neoplasias, como melanoma avançado, câncer de pulmão, carcinoma de células renais, câncer de bexiga e Linfoma de Hodgkin $(4,15,25)$. Testes 
clínicos em malignidades sólidas nos quais foram empregados os anticorpos tiveram início em 2012 (25). No primeiro teste clínico realizado com anticorpo monoclonal PD-1 (IgG4 totalmente humano) foi observada a regressão de alguns tumores com respostas parciais e uma resposta completa $(4,22)$. Os tumores que expressam PD-1, como de cólon, renal, de pulmão e melanoma apresentaram regressão mais importante com o emprego dos anticorpos monoclonais $(22,26)$. No carcinoma de cólon retal e no melanoma, ocorreu alteração drástica no índice de mutação, de forma que o FDA liberou o Nivolumab em 2014, sendo registrado como o primeiro anticorpo monoclonal anti-PD-1 no tratamento do melanoma, ensejando testes em outros tipos de tumores (25).

A farmacocinética do Nivolumab é linear e os testes foram realizados com concentrações que variaram de 01 a $10 \mathrm{mg} / \mathrm{kg}$. Foi constatado que o clearance (CL) é de $9,5 \mathrm{~mL} / \mathrm{h}$ e que o volume de distribuição no estado estacionário foi de $8 \mathrm{~L}$. Por se tratar de anticorpo monoclonal, a via metabólica ocorre por catabolismo, com degradação em peptídeos e aminoácidos (27).

A farmacocinética foi avaliada em estudos de fase I, fase II e fase III em diferentes pacientes, com diferentes tipos de tumores. Os resultados indicaram que após a administração por via intravenosa, o Nivolumab sofre bidistribuição, uma rápida e outra tardia. A última mostrou um tempo de meia-vida de 25 a 26 dias (2).

Quanto ao clearance, foi observado que aumenta de forma proporcional ao aumento do peso corporal. $\mathrm{O}$ estado de equilíbrio de concentração foi mantido em estado mínimo de forma uniforme frente à variedade de pesos corpóreos (27).

O Nivolumab foi submetido a estudos que avaliaram seu efeito em pacientes com comprometimento renal leve, médio e grave e pacientes hepáticos com comprometimento leve. Não foram relatadas alterações clinicamente significativas de comprometimento renal e/ou hepático com o uso do fármaco, indicando segurança no tratamento, embora maior atenção no acompanhamento clínico seja necessária (27).

O primeiro estudo realizado com o Nivolumab em portadores de $\mathrm{LH}$, contou com 23 pacien- tes com histórico de recidiva ou doença refratária $(4,6)$. No estudo de fase I os pacientes receberam $3 \mathrm{mg} / \mathrm{kg}$ do Nivolumab a cada 15 dias, até que se obtivesse resposta clínica de progressão ou regressão da doença ou ainda toxicidade inaceitável. Dez pacientes apresentaram respostas duráveis durante o tratamento, quatro tiveram progressão da doença, cinco interromperam o tratamento para serem submetidos ao transplante de células-tronco e somente um paciente interrompeu o tratamento por reações adversas relacionadas ao medicamento (6). O tratamento com Nivolumab proporciona resposta clínica eficiente se houver uma resposta imune prévia, como em pacientes LH reincidentes e refratários, uma vez que a via PD-1 não está envolvida na ativação direta das células efetoras $(4,28)$.

Outros ensaios clínicos investigaram a eficácia do Nivolumab como tratamento em pacientes com LH reincidentes ou refratários. Um estudo multicêntrico avaliou a terapia combinada de Brentuximab, Vedotin e Nivolumab em sessenta e dois pacientes, mostrando uma taxa de resposta complete, entre todos os pacientes tratados, de $61 \%$. A combinação desses fármacos resultou em diminuição do volume do tumor e da atividade metabólica de $98 \%$ e $93 \%$ dos pacientes avaliados pela eficácia, respectivamente (17).

Estudos apontam que o emprego do Nivolumab em pacientes portadores de LH produziu uma resposta de $65 \%$ na remissão do tumor, tornando os fármacos anti-PD-1 como opções para tratamento da patologia (29)relapse is still frequent. The programmed cell death protein 1 (PD-1.

Deve-se considerar, no entanto, que os pacientes podem ser tratados por períodos de meses ou anos, embora os efeitos de longo prazo do Nivolumab sejam desconhecidos, bem como a duração da sua resposta (6).

Reações adversas do Nivolumab. Os estudos sobre o anti-PD-1 Nivolumab mostraram nível de segurança aceitável quando comparado com a quimioterapia clássica (7). Como a terapia consiste no bloqueio da via coinibitória de tolerância, os efeitos adversos estão relacionados à autoimunidade, sendo caracterizados como Eventos Adversos Relacionados à Imunidade - IRAE (Immunity-Related Adverse Events). Esses variam, podendo ocorrer 
colite, hepatite, dermatite, pneumonite e distúrbios endócrinos como na hipófise e tireoide $(4,26,30$ 32). Para tratar tais distúrbios são empregados corticosteroides, inibidores de TNF ou outra terapia imunossupressora (26,33).

As IRAE são mais frequentes em malignidades hematológicas do que em tumores sólidos. O fundamento para isso ainda não está claro, mas não pode ser negligenciado o fato de que esses pacientes são submetidos a outros tratamentos previamente, o que contribui para que o hospedeiro se torne mais debilitado e mais susceptível a efeitos adversos. Em estudo realizado em portadores LH refratário, 22 dos 23 pacientes estudados apresentaram reações adversas em vários graus, sendo as mais comuns erupções cutâneas (22\%), fadiga (13\%), náusea $(13 \%)$, diarreia (12\%) e reações em virtude da infusão (9-20\%), e um paciente apresentou síndrome mielodisplásica. O último foi submetido a seis tipos de tratamentos prévios, que envolveram quimioterapia, radioterapia e transplante de células-tronco. Não houve mortes durante o estudo, ou reações de grau 4, segundo a classificação dos critérios do Instituto Nacional do Câncer para Eventos Adversos (Common Terminology Criteria for Adverse Events) (6,33).

Em outro estudo de fase II, realizado com 80 pacientes portadores de LH recidivante ou refratário, 71 pacientes $(89 \%)$ apresentaram IRAE, sendo as mais comuns fadiga (25\%) (31), reação em virtude da infusão (20\%), erupção cutânea (15\%) e diarreia (10\%). Reações de grau 3 foram relatadas por 17 pacientes $(21 \%)$ e 3 pacientes com reação de grau 4 (4\%) (6). As reações adversas hepáticas foram relatadas em $5 \%$ dos casos, com aumento de aspartato aminotransferase (AST) (28\%), aumen- to de alanina aminotransferase (ALT) (18\%) e de fosfatase alcalina $(22 \%)(23,19)$. Considerando as reações adversas renais, houve aumento de creatinina sérica (13\%). Desde o início dos testes com o Nivolumab não foram registradas variações quanto aos tipos de efeitos adversos (34).

\section{CONCLUSÃO}

Aproximadamente $15 \%$ dos pacientes portadores de LH tratados com quimioterapia convencional apresentam recidiva e duração do período de remissão reduzida a cada novo tratamento. Além disso, esse tratamento também está relacionado à ocorrência de inúmeros efeitos adversos.

O tratamento ideal de LH deve induzir resposta duradoura e com a maior taxa de sobrevida. O bloqueio dos pontos de controle apresenta boas perspectivas no tratamento de doenças hematológicas malignas, com menos efeitos adversos graves quando comparado à quimioterapia clássica. Nesse contexto, recentemente foi introduzido o emprego de terapias que fortalecem o sistema imunológico aumentando a resposta antitumoral, realizado com anticorpos monoclonais anti-PD-1, como o Nivolumab.

Dessa forma, apesar de ser necessário o desenvolvimento de novos estudos envolvendo o uso de anticorpos monoclonais anti-PD-1 a fim de elucidar outros aspectos do tratamento antitumoral, garantindo melhor compreensão das particularidades e proporcionando maior segurança ao paciente, essa abordagem é promissora e provavelmente será priorizada nos próximos anos, podendo ser um caminho importante inclusive na abordagem de outros tipos de tumores.

\section{REFERÊNCIAS}

1. Rodrigues AB, Martin LGR, Moraes MW. Oncologia Multiprofissinal: patologias, assistência e gerenciamento. Walksman RD, Farah OGD (org). Barueri, SP: Editora Manole; 2016.

2. Bajaj G, Wang X, Agrawal S, Gupta M, Roy A, Feng Y. Model-Based Population Pharmacokinetic Analysis of
Nivolumab in Patients With Solid Tumors. CPT Pharmacometrics Syst Pharmacol. 2016;4. DOI: 10.1002/ psp4.12143.

3. Rizzatti EG. Linfoma de Hodgkin versus linfoma não Hodgkin: qual é a diferença? Fleury Medicina e Saúde. $2011 ; 1-3$. 
4. Ansell SM, Lesokhin AM, Borrello I, Halwani A, Scott EC, Gutierrez M, et al. PD-1 Blockade with Nivolumab in Relapsed or Refractory Hodgkin's Lymphoma. N Engl J Med. 2015;372(4):311-319. DOI: 10.1056/NEJMoa1411087.

5. Barrett MT, Anderson KS, Lenkiewicz E, Andreozzi M, Cunliffe HE, Klassen CL, et al. Genomic amplification of 9p24.1 targeting JAK2, PD-L1, and PD-L2 is enriched in high-risk triple negative breast cancer. Oncotarget. 2015;6(28):26483-26493. DOI: 10.18632/oncotarget.4494.

6. Bond DA, Alinari L. Emerging treatment options for the management of Hodgkin's lymphoma: clinical utility of nivolumab. J Blood Med. 2017;8:41-54. DOI 10.2147/ JBM.S117452.

7. Bryan LJ, Gordon LI. Blocking tumor escape in hematologic malignancies: The anti-PD-1 strategy. Blood Rev. 2015;29(1):25-32. DOI: 10.1016/j.blre.2014.09.004.

8. Guimarães JRQ. Linfoma de Hodgkin. In: Guimarães JRQ. Manual de Oncologia. $2^{\circ}$ ed. 2004. 27-36 p.

9. Connors JM. State-of-the-art therapeutics: Hodgkin's lymphoma. J Clin Oncol. 2005;23(26):6400-6408. DOI: 10.1200/JCO.2005.05.016.

10. Devita VT, Serpick AA, Carbone PP. Combination Chemotherapy in the Treatment of Advanced Hodgkin's Disease. Ann Intern Med. 1970;73(6):881. DOI: 10.7326/0003-4819-73-6-881.

11. Bonadonna G, Zucali R, Monfardini S, De Lena M, Uslenghi C. Combination chemotherapy of Hodgkin's disease with adriamycin, bleomycin, vinblastine, and imidazole carboxamide versus MOPP. Cancer.1975;36(1):252-9. DOI: $10.1002 / 1097-0142(197507) 36: 1<252:: A I D-C N-$ CR2820360128>3.0.CO;2-7.

12. Canellos GP, Anderson JR, Propert KJ, Nissen N, Cooper MR, Henderson ES, et al. Chemotherapy of Advanced Hodgkin's Disease with MOPP, ABVD, or MOPP Alternating with ABVD. N Engl J Med. 1992;327(21):147884. DOI: 10.1056/NEJM199211193272102.

13. Costa G, Lopes B, Moreira WB, Soares AN. Avaliação dos resultados do tratamento de pacientes portadores de linfoma de Hodgkin com esquema ABVD em primeira linha. Rev Bras Oncol Clínica. 2011; 8(29): 112-120.

14. Vardhana S, Younes A. The immune microenvironment in Hodgkin lymphoma: T cells, B cells, and immune checkpoints. Haematologica. 2016;101(7):794-802. DOI: $10.3324 /$ haematol.2015.132761.

15. Guo L, Zhang H, Chen B. Nivolumab as Programmed Death-1 (PD-1) Inhibitor for Targeted Immunotherapy in Tumor. J Cancer. 2017;8(3):410-6. DOI: 10.7150/ jca. 17144

16. Zitvogel L, Kroemer G. Targeting PD-1/PD-L1 interactions for cancer immunotherapy. Oncoimmunology. 2012;1(8):1223-5. DOI: 10.4161/onci.21335

17. Herrera AF, Moskowitz AJ, Bartlett NL, Vose JM, Ramchandren R, Feldman TA, et al. Interim results of brentuximab vedotin in combination with nivolumab in patients with relapsed or refractory Hodgkin lymphoma. Blood. 2018;131: 1183-1194. DOI: 10.1182/ blood-2017-10-811224.

18. Onizuka M, Kojima M, Matsui K, Machida S, Toyosaki M, Aoyama Y, et al. Successful treatment with low-dose nivolumab in refractory Hodgkin lymphoma after allogeneic stem cell transplantation. Int J Hematol. 2017;106(1):141145. DOI: $10.1007 / \mathrm{s} 12185-017-2181-9$.

19. Bour-Jordan H, Esensten JH, Martinez-Llordella M, Penaranda C, Stumpf M, Bluestone JA. Intrinsic and extrinsic control of peripheral T-cell tolerance by costimulatory molecules of the CD28/B7 family. Immunol Rev. 2011; 241:180-205. DOI: 10.1111/j.1600-065X.2011.01011.x.

20. Jacobsen ED. Restoring antitumor immunity via PD-1 blockade after autologous stem-cell transplantation for diffuse large B-cell lymphoma. J Clin Oncol. 2013 ;31(33):4268-4270. DOI: 10.1200/JCO.2013.51.7680.

21. Good-Jacobson KL, Szumilas CG, Chen L, Sharpe AH, Tomayko MM, Shlomchik MJ. PD-1 regulates germinal center B cell survival and the formation and affinity of long-lived plasma cells. Nat Immunol. 2010 ;11(6):535542. DOI: $10.1038 /$ ni.1877.

22. Pardoll DM. The blockade of immune checkpoints in cancer immunotherapy. Nat Rev Cancer. 2012;12(4):252264. DOI: $10.1038 / \mathrm{nrc} 3239$.

23. Galanina N, Kline J, Bishop MR. Emerging role of checkpoint blockade therapy in lymphoma. Ther Adv Hematol. 2017;8(2):81-90. DOI: $10.1177 / 2040620716673787$.

24. Lorenz U. SHP-1 and SHP-2 in T cells: two phosphatases functioning at many levels. Immunol Rev. 2009 [;228(1):342-59. DOI: 10.1111/j. 1600-065X.2008.00760.x

25. Sunshine J, Taube JM. PD-1/PD-L1 inhibitors. Curr Opin Pharmacol. 2015;23:32-38. DOI: 10.1016/j. coph.2015.05.011.

26. Postow MA, Callahan MK, Wolchok JD. Immune Checkpoint Blockade in Cancer Therapy. J Clin Oncol. 2015;33(17):1974-1982. DOI: 10.1200/ JCO.2014.59.4358. 
27. Bristol-Myers Squibb Farmacêutica LTDA. Bula Opdivo [Internet]. Rev0418, 2018. p. 87. Available at: https:// www.bms.com/assets/bms/brazil/documents/Opdivo SOL_INJ_VPS_Rev0418.pdf

28. Corsello SM, Barnabei A, Marchetti P, De Vecchis L, Salvatori R, Torino F. Endocrine Side Effects Induced by Immune Checkpoint Inhibitors. J Clin Endocrinol Metab. 2013;98(4):1361-1375. DOI: 10.1210/jc.2012-4075

29. Herbaux C, Gauthier J, Brice P, Drumez E, Ysebaert L, Doyen $\mathrm{H}$, et al. Efficacy and tolerability of nivolumab after allogeneic transplantation for relapsed Hodgkin lymphoma. Blood. 2017;129(18):2471-2478. DOI: 10.1182/ blood-2016-11-749556.

30. Haanen JBAG, Thienen $\mathrm{H}$ van, Blank CU, Powrie F, Izcue $\mathrm{A}$, et al. Toxicity patterns with immunomodulating antibodies and their combinations. Semin Oncol. 2015;42(3):423-428. DOI: $10.1053 /$ j.seminoncol.2015.02.011.
31. Naidoo J, Page DB, Li BT, Connell LC, Schindler K, Lacouture ME, et al. Toxicities of the anti-PD-1 and anti-PD-L1 immune checkpoint antibodies. Ann Oncol. 2015;26(12): 2375-2391. DOI: 10.1093/annonc/mdv383.

32. Tardy MP, Gastaud L, Boscagli A, Peyrade F, Gallamini A, Thyss A. Autoimmune hemolytic anemia after nivolumab treatment in Hodgkin lymphoma responsive to immunosuppressive treatment. A case report. Hematol Oncol. 2017;35(4):875-877. DOI: 10.1002/hon.2338.

33. Brahmer JR, Tykodi SS, Chow LQM, Hwu W-J, Topalian SL, Hwu P, et al. Safety and activity of anti-PD-L1 antibody in patients with advanced cancer. N Engl J Med. 2012;366(26):2455-2465. DOI: 10.1056/NEJMoa1200694.

34. Nivolumab (Professional Patient Advice) - Drugs.com.. Available at: https://www.drugs.com/ppa/nivolumab. html 